\title{
LABORATORY SPECTROSCOPY OF PROTONATED PAH MOLECULES RELEVANT FOR INTERSTELLAR CHEMISTRY
}

\author{
O. Dopfer ${ }^{1}$
}

\begin{abstract}
In this contribution, we summarize the recent progress made in recording laboratory infrared (IR) spectra of protonated polycyclic aromatic hydrocarbon molecules $\left(\mathrm{H}^{+} \mathrm{PAH}\right)$ in the gas phase. The IR spectra of a large variety of $\mathrm{H}^{+} \mathrm{PAH}$ species ranging from benzene to coronene have been obtained by various variants of photodissociation spectroscopy. The employed techniques include single-photon IR photodissociation (IRPD) of tagged $\mathrm{H}^{+} \mathrm{PAH}$ ions and IR multiple-photon dissociation (IRMPD) of bare $\mathrm{H}^{+} \mathrm{PAH}$ ions. The comparison of the laboratory IR spectra with astronomical spectra supports the hypothesis that $\mathrm{H}^{+} \mathrm{PAH}$ ions are possible carriers of the unidentified IR emission (UIR) bands. Moreover, the spectra provide detailed information about the geometric and electronic structure as well as the chemical reactivity and stability of these fundamental hydrocarbon ions.
\end{abstract}

\section{Introduction}

The unidentified infrared (UIR) emission bands are observed in a great variety of galactic and extragalactic sources. They are currently attributed to IR fluorescence of UV-excited polycyclic aromatic hydrocarbon (PAH) molecules and their more complex derivatives, such as ionic PAH, (de-)hydrogenated and methyl-substituted PAH, PAH clusters, nitrogen- and silicon-containing PAH, and PAH-metal ion adducts (Léger \& Puget 1984; Allamandola et al. 1985; Tielens 2008). Recently, also protonated $\mathrm{PAH}$ molecules, $\mathrm{H}^{+} \mathrm{PAH}$, have been suggested because laboratory experiments demonstrated that they can readily be formed through efficient protonation of neutral $\mathrm{PAH}$ or attachment of $\mathrm{H}$-atoms to $\mathrm{PAH}^{+}$radical cations (Snow et al. 1998). In addition, their calculated IR spectra identify $\mathrm{H}^{+} \mathrm{PAH}$ as promising candidates for UIR band carriers (Hudgins et al. 2001).

In order to test the $\mathrm{H}^{+} \mathrm{PAH}$ hypothesis, laboratory IR spectra of $\mathrm{H}^{+} \mathrm{PAH}$ are required for comparison with astronomical UIR spectra. Such spectra have,

1 Institut für Optik und Atomare Physik, Technische Universität Berlin, Hardenbergstrasse 36, 10623 Berlin, Germany; e-mail: dopfer@physik.tu-berlin.de 
however, been lacking until recently (Lorenz et al. 2007), largely due to experimental challenges involved in the generation of high abundances sufficient for spectroscopic interrogation. Recent progress in the development of efficient ion sources and sensitive IR spectroscopic techniques have allowed to obtain the first IR spectra of size-selected protonated aromatic molecules and their weakly-bound clusters in the gas phase by coupling tandem mass spectrometry with resonant vibrational photodissociation spectroscopy (Solca \& Dopfer 2001; Solca \& Dopfer 2003a). The application of these techniques to protonated aromatic molecules has been reviewed previously (Dopfer 2006). In this contribution, we summarize the results obtained in the past decade for a large variety of $\mathrm{H}^{+} \mathrm{PAH}$ ions, ranging from protonated benzene to protonated coronene.

\section{Experimental techniques}

Several experimental approaches have been used to generate isolated and microsolvated $\mathrm{H}^{+} \mathrm{PAH}$ cations in the gas phase for spectroscopic interrogation. Ion and cluster ion generation has been accomplished in an electron-impact or dischargedriven supersonic plasma expansion. This approach was used to produce protonated benzene and naphthalene, $\mathrm{C}_{6} \mathrm{H}_{7}^{+}$and $\mathrm{C}_{10} \mathrm{H}_{9}^{+}$, and their weakly-bound clusters with ligands $\mathrm{L}=\mathrm{Ar}, \mathrm{N}_{2}, \mathrm{CH}_{4}$, and $\mathrm{H}_{2} \mathrm{O}$ (Solca \& Dopfer 2002; Solca \& Dopfer 2003b; Douberly et al. 2008; Ricks et al. 2009). Alternatively, isolated $\mathrm{H}^{+} \mathrm{PAH}$ can be generated by chemical ionization of $\mathrm{PAH}$ in an ion cyclotron resonance (ICR) mass spectrometer using protonating agents, such as $\mathrm{CH}_{5}^{+}$or $\mathrm{C}_{2} \mathrm{H}_{5}^{+}$, as successfully demonstrated for $\mathrm{C}_{6} \mathrm{H}_{7}^{+}$and $\mathrm{C}_{10} \mathrm{H}_{9}^{+}$(Jones et al. 2003; Dopfer et al. 2005; Lorenz et al. 2007). Larger PAH molecules have low vapour pressure and a feasible option to generate sufficient abundances of their protonated species is electrospray ionization. This technique has been applied to characterize larger $\mathrm{H}^{+} \mathrm{PAH}$ ions (Knorke et al. 2009), ranging from protonated anthracene, $\mathrm{C}_{14} \mathrm{H}_{11}^{+}$, to protonated coronene, $\mathrm{C}_{24} \mathrm{H}_{13}^{+}$.

Two major spectroscopic strategies have successfully been employed to obtain IR spectra of isolated and microsolvated $\mathrm{H}^{+} \mathrm{PAH}$ ions. The first technique couples modern low-intensity optical parametric oscillator (OPO) laser systems, typically operating in the 1000-4000 $\mathrm{cm}^{-1}$ range, with tandem mass spectrometers to record single-photon IRPD spectra of cold $\mathrm{H}^{+} \mathrm{PAH}-\mathrm{L}_{m}$ cluster ions by monitoring the loss of weakly-bound ligands L (Solca \& Dopfer 2002; Solca \& Dopfer 2003b; Douberly et al. 2008; Ricks et al. 2009). The low intensities of OPO lasers are usually insufficient to drive multiple-photon processes. The influence of the ligands on the IR spectrum of the central $\mathrm{H}^{+} \mathrm{PAH}$ ion can usually be neglected for weakly-bound ligands (e.g., Ar), i.e. the IRPD spectra of the tagged species correspond to a very good approximation (to within a few $\mathrm{cm}^{-1}$ ) to the IR spectra of the bare ion. Moreover, the clusters are cold as they are produced in molecular beams. As a result of the low internal temperature (typically well below $50 \mathrm{~K}$ ) and the single-photon dissociation process, IRPD spectra display high resolution and sharp transitions with widths of the order of $5 \mathrm{~cm}^{-1}$. Moreover, they display high sensitivity, i.e. even transitions with low IR activity can readily be detected. 
The IRPD approach has been applied to obtain the first IR spectrum of $\mathrm{C}_{6} \mathrm{H}_{7}^{+}$in the gas phase (Solca \& Dopfer 2002).

The second spectroscopic strategy couples high-intensity free electron laser (FEL) sources (FELIX and CLIO), operating in the complementary $50-2500 \mathrm{~cm}^{-1}$ range, with ion cyclotron resonance (ICR) mass spectrometry to drive IRMPD of isolated, strongly-bound $\mathrm{H}^{+} \mathrm{PAH}$ by monitoring either $\mathrm{H}$-atom or $\mathrm{H}_{2}$ loss (Jones et al. 2003; Dopfer et al. 2005; Lorenz et al. 2007; Zhao et al. 2009; Knorke et al. 2009). In general, the spectral resolution observed in the IRMPD spectra (circa $30 \mathrm{~cm}^{-1}$ ) is lower than in IRPD spectra due to the finite bandwidth of the FEL, the broader rotational contour (as the ions probed in the ICR cell are at room temperature), spectral congestion due to overlapping transitions, and spectral broadening arising from the multiple-photon character of the IRMPD process. Typically, several tens of IR photons are required to drive the IRMPD process due to the large dissociation energies for $\mathrm{H}$ or $\mathrm{H}_{2}$ elimination from $\mathrm{H}^{+} \mathrm{PAH}$. As a further consequence of the IRMPD mechanism, weakly IR active transitions with cross sections below a certain threshold are not observed. The IRMPD approach has been utilized to obtain the first IR spectrum of an isolated $\mathrm{H}^{+} \mathrm{PAH}$, namely $\mathrm{C}_{10} \mathrm{H}_{9}^{+}$(Lorenz et al. 2007). Figure 1 compares the IRMPD spectra of a variety of $\mathrm{H}^{+} \mathrm{PAH}$ obtained so far, including protonated benzene, naphthalene, anthracene, tetracene, pentacene, azulene, perylene, pyrene, coronene, acenaphthene, and acenaphthylene. While this contribution focuses on the general appearance of the IR spectra and the comparsion with an UIR spectrum representative of a highlyionized region of the interstellar medium (Tielens 2008; Fig. 1), a full account of the potential energy surfaces with structural and vibrational analyses is given elsewhere (Zhao et al. 2009; Zhao 2010). Significantly, apart from benzene $\mathrm{H}^{+}$, the IRMPD spectra in Figure 1 correspond to the first spectroscopic data of these $\mathrm{H}^{+} \mathrm{PAH}$ in the gas phase, providing very valuable information about the geometric and electronic structures as well as the chemical reactivity and stability of these fundamental ions. For an illustration of the information content of these spectra, the reader is referred to a recent analysis of the azuleneH ${ }^{+}$spectrum using quantum chemical calculations (Zhao et al. 2009). A similar analysis was performed for all $\mathrm{H}^{+} \mathrm{PAH}$ spectra in Figure 1 (Zhao 2010) and will be published elsewhere.

\section{Results and discussion}

Initial IRPD spectra obtained for $\mathrm{C}_{6} \mathrm{H}_{7}^{+}$in the $\mathrm{CH}$ stretch range using the tagging technique (Solca \& Dopfer 2002; Solca \& Dopfer 2003b) demonstrated for the first time spectroscopically that the proton is attached to a $\mathrm{C}$-atom ( $\sigma$-complex) rather than the $\pi$ electron system of the aromatic ring ( $\pi$-complex), a conclusion that also holds for larger $\mathrm{H}^{+} \mathrm{PAH}$. In addition, these spectra do not show coincidences with prominent UIR bands. Subsequent IRMPD spectra reported for isolated $\mathrm{C}_{6} \mathrm{H}_{7}^{+}$ in the fingerprint range (Fig. 1) using CLIO and monitoring $\mathrm{H}_{2}$ loss (Jones et al. 2003; Dopfer et al. 2005) and higher-resolution IRPD spectra of Ar-tagged $\mathrm{C}_{6} \mathrm{H}_{7}^{+}$ ions (Douberly et al. 2008) confirm these initial conclusions. 

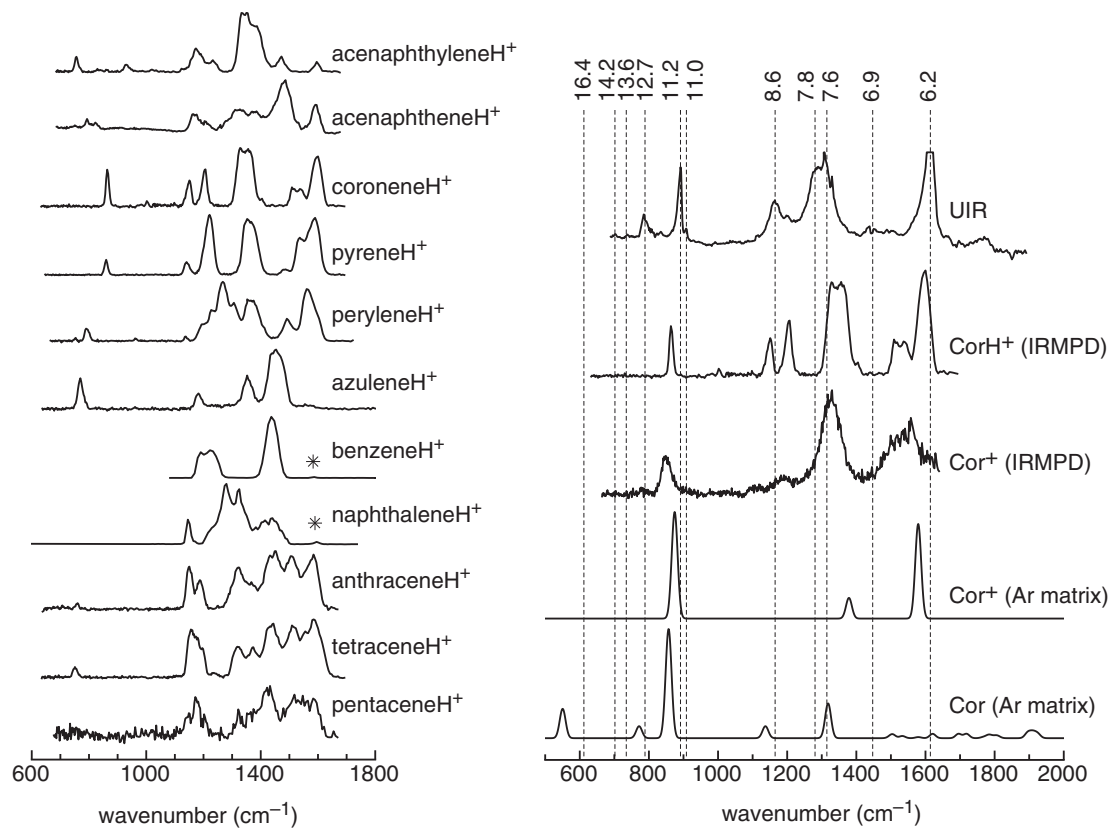

Fig. 1. Left: IRMPD spectra of a variety of $\mathrm{H}^{+} \mathrm{PAH}$ ions (Dopfer 2005; Lorenz et al. 2007; Dopfer 2008; Knorke et al. 2009; Zhao et al. 2009); the spectra of benzene $\mathrm{H}^{+}$ and naphthalene $\mathrm{H}^{+}$were recorded using CLIO, whereas all other spectra were obtained using FELIX; bands marked by asterisks are largely suppressed due to reduced laser power. Right: IR spectra of coronene, coronene ${ }^{+}$and coronene $\mathrm{H}^{+}$compared to an UIR spectrum (Knorke et al. 2009). The dashed lines indicate positions of UIR bands in $\mu \mathrm{m}$.

Next, IRMPD spectra were recorded for $\mathrm{C}_{10} \mathrm{H}_{9}^{+}$, the smallest $\mathrm{H}^{+} \mathrm{PAH}$, using CLIO and the major conclusions can be summarized as follows (Lorenz et al. 2007). First, the IRMPD spectra of $\mathrm{C}_{6} \mathrm{H}_{7}^{+}$and $\mathrm{C}_{10} \mathrm{H}_{9}^{+}$are very different (Fig. 1), implying a strong dependence of the IR spectra of $\mathrm{H}^{+} \mathrm{PAH}$ on the number of aromatic rings, at least for small PAH. Second, the IRMPD spectrum of $\mathrm{C}_{10} \mathrm{H}_{9}^{+}$ demonstrates striking coincidences with the UIR spectrum. Both, the positions and IR intensities of the $\mathrm{C}_{10} \mathrm{H}_{9}^{+}$bands match the astronomical spectrum much better than the IR spectra of neutral and ionized naphthalene. These important conclusions were later confirmed by IRPD spectra of $\mathrm{C}_{10} \mathrm{H}_{9}^{+}-\mathrm{Ar}$ (Ricks et al. 2009).

The close match of the $\mathrm{C}_{10} \mathrm{H}_{9}^{+}$and UIR spectra called for laboratory IR spectra of larger $\mathrm{H}^{+} \mathrm{PAH}$, in order to follow their evolution as a function of PAH size. This was also of importance in the light of astrochemical models, which suggest PAH molecules consisting of 20-80 C-atoms to be photochemically most stable in interstellar clouds (Tielens 2008). To this end, IRMPD spectra of a variety of larger $\mathrm{H}^{+} \mathrm{PAH}$ species (Fig. 1) have been recorded using FELIX (Dopfer 2008). 
Figure 1 compares the IRMPD spectra of linear catacondensed $\mathrm{H}^{+} \mathrm{PAH}$ featuring $n$ aromatic rings with $n=1-5$ (Knorke et al. 2009). The $n=1$ and $n=2$ spectra are significantly different in appearance, and both differ from those for $n=3-5$. However, the IRMPD spectra for $n=3-5$ are rather similar, suggesting convergence of the spectral evolution at around $n=3$. Comparison of the $n=3-5$ spectra with an UIR spectrum representative of a highly-ionized region of the interstellar medium (Tielens 2008; Fig. 1) reveals good correspondence for transitions assigned to in-plane $\mathrm{CH}$ bend and $\mathrm{CC}$ stretch modes. In addition, the highest-frequency $\mathrm{CC}$ stretch mode at around $1600 \mathrm{~cm}^{-1}$ approaches the $6.2 \mu \mathrm{m}$ UIR feature with increasing $n$. The intense, lower-frequency CC stretch modes at $1450 \mathrm{~cm}^{-1}$ are characteristic of all linear catacondensed $\mathrm{H}^{+} \mathrm{PAH}$ and correlate with the $6.9 \mu \mathrm{m}$ UIR feature, which is however only weakly observed in the astronomical spectrum. Similar conclusions apply to the $1500 \mathrm{~cm}^{-1}$ transition.

Not only are linear catacondensed $\mathrm{H}^{+} \mathrm{PAH}$ with $n=1-5$ probably too small to be photostable, they are also less stable than pericondensed two-dimensional $\mathrm{H}^{+} \mathrm{PAH}$. To this end, we recorded also the IRMPD spectrum of protonated coronene $\left(\mathrm{C}_{24} \mathrm{H}_{13}^{+}\right)$, a pericondensed $\mathrm{PAH}$, which is often considered as the prototypical and representative $\mathrm{PAH}$ molecule present in the interstellar medium. Figure 1 compares the available experimental IR spectra for different degrees of ionization and protonation of coronene with the UIR spectrum (Knorke et al. 2009). Comparison of the spectra of coronene and coronene ${ }^{+}$recorded in an Ar matrix demonstrates the significant impact of ionization on the IR intensities. The coronene ${ }^{+}$spectrum recorded in the cold Ar matrix and via IRMPD in the gas phase are similar but illustrate the typical redshift and broadening of bands induced by the IRMPD process. The IRMPD spectrum of coronene ${ }^{+}$and coroneneH $^{+}$are again similar. The redshifts and broadening effects due to IRMPD are, however, less pronounced for the protonated species due to its lower dissociation energy. Moreover, the bands in the $1100-1200 \mathrm{~cm}^{-1}$ range display higher IR activity. Significantly, the intense CC stretch band at $1600 \mathrm{~cm}^{-1}(6.25 \mu \mathrm{m})$ occurs at a higher frequency than for the radical cation and closely approaches the $6.2 \mu \mathrm{m}$ band observed in the UIR spectrum.

As the UIR bands are caused by IR fluorescence of vibrationally hot species, the vibrational transitions observed are shifted in frequency from the frequencies of fundamental transitions due to vibrational anharmonicities. Similarly, the multiple-photon nature of the IRMPD process also shifts the frequencies to lower values in comparison to fundamental transitions, again via the effect of anharmonicities. Thus, it is indeed meaningful to compare IRMPD spectra with the UIR bands, as both processes and the resulting IR spectra are affected by the same type of vibrational anharmonicity (Oomens et al. 2003). In view of this argument, the good agreement between the IRMPD spectrum of coroneneH $^{+}$and the UIR spectrum in Figure 1 provides for the first time compelling experimental evidence for the hypothesis that $\mathrm{H}^{+} \mathrm{PAH}$ indeed contribute to the UIR spectrum. Future efforts include recording IR spectra of even larger $\mathrm{H}^{+} \mathrm{PAH}$ ions in order to follow the spectral evolution as a function of PAH size. In addition, electronic spectra of $\mathrm{H}^{+} \mathrm{PAH}$ will be recorded, as they are promising candidates for carriers 
of the diffuse interstellar bands (Pathak \& Sarre 2008; see also Hammonds et al., elsewhere in this volume). First encouraging results have been obtained for $\mathrm{C}_{10} \mathrm{H}_{9}^{+}$ (Alata et al. 2010), which strongly absorbs in the visible range, supporting the hypothesis that larger $\mathrm{H}^{+} \mathrm{PAH}$ ions may indeed be carriers of some of the diffuse interstellar bands.

This work was supported by TU Berlin, the Deutsche Forschungsgemeinschaft (DO 729/2; 729/3), and the European Community - Research Infrastructure Action under the FP6 Structuring the European Research Area Program (through the integrated infrastructure initiative Integrating Activity on Synchrotron and Free Electron Laser Science).

\section{References}

Allamandola, L.J., Tielens, A.G.G.M., \& Barker, J.R., 1985, ApJ, 290, L25

Alata, I., Omidyan, R., Broquier, M., et al., 2010, Phys. Chem. Chem. Phys., 12, 14456

Dopfer, O., Solca, N., Lemaire, J., et al., 2005, J. Phys. Chem. A, 109, 7881

Dopfer, O., 2006, J. Phys. Org. Chem., 19, 540

Dopfer, O., 2008, report for project FELIX-030

Douberly, G.E., Ricks, A.M., Schleyer, P.V.R., \& Duncan, M.A., 2008, J. Phys. Chem. A, 112,4869

Hudgins, D.M., Bauschlicher, C.W., \& Allamandola, L.J., 2001, Spectrochim. Acta Part A, 57,907

Jones, W., Boissel, P., Chiavarino, B., et al., 2003, Angew. Chem. Int. Ed., 42, 2057

Knorke, H., Langer, J., Oomens, J., \& Dopfer, O., 2009, ApJ, 706, L66

Léger, A., \& Puget, J.L., A\&A, 137, L5

Lorenz, U.J., Solca, N., Lemaire, J., Maitre, P., \& Dopfer, O., 2007, Angew. Chem. Int. Ed., 46, 6714

Pathak, A., \& Sarre, P.J., 2008, MNRAS, 391, L10

Ricks, A.M., Douberly, G.E., \& Duncan, M.A., 2009, ApJ, 702, 301

Snow, T.L., Page, L.V., Keheyan, Y., \& Bierbaum, V.M., 1998, Nature, 391, 259

Solca, N., \& Dopfer, O., 2001, Chem. Phys. Lett., 342, 191

Solca, N., \& Dopfer, O., 2002, Angew. Chem. Int. Ed., 41, 3628

Solca, N. \& Dopfer, O., 2003, J. Am. Chem. Soc., 125, 1421

Solca, N., \& Dopfer, O., 2003, Chem. Eur. J., 9, 3154

Tielens, A.G.G.M., 2008, ARA\&A, 46, 337

Oomens, J., Tielens, A.G.G.M., Sartakov, B.G., von Helden, G., \& Meijer, G., 2003, ApJ, 591, 968

Zhao, D., Langer, J., Oomens, J., \& Dopfer, O., 2009, J. Chem. Phys., 131, 184307

Zhao, D., 2010, Diploma Thesis, TU Berlin, Germany 